\title{
THE PROPER MOTIONS OF SUNSPOTS AND THE MAGNETIC FIELD OF ACTIVE REGIONS
}

\author{
G.V. KUKLIN \\ (Siberian Institute of Terrestrial Magnetism, \\ Ionosphere and Radio Propagation, Academy of \\ Sciences, Irkutsk, U.S.S.R.)
}

According to our program of sunspot proper motion investigations (Kuklin and Syklen, 1966) we study the interdependence of the sunspot proper motions inside the group and the magnetic field of the whole group or active region. This aspect of the dynamics of matter in disturbed regions of the Sun was not considered practically up to the last time.

Bumba (1964) came to the conclusion that the individual small umbrae move practically parallel to the magnetic field isolines (of the longitudinal component) and that the general character of the magnetic-field distribution in the active region changes very little during the motions. The magnetic field creeps in the active region according to many recent investigations of the magnetic-field configuration in active regions (for example Stepanov et al., 1967). Therefore the small umbrae moving along the magnetic-field isolines must cross the magnetic lines of force quite perpendicularly, which is unlikely to be in accordance with the magnetohydrodynamics.

Gopasyuk and Moreton (1965) found that the transverse component of the magnetic field changes correspondingly to the main sunspot proper motions: the main sunspots pull out the magnetic lines of force during their motions and deform the magnetic field in such a way.

We have studied the proper motions in three sunspot groups during some days, taking into account the magnetic-field observations (three components) with the help of the solar magnetograph. We came to the following conclusions:

(1) The main sunspots in the active region move independently of the active region magnetic field but pulling the magnetic lines of force hardly fastened together and changing the magnetic field correspondingly.

(2) The satellites of the main sunspots (small umbrae and penumbra bits) accompany the main sunspots irrespectively of the magnetic field in general as if crossing the magnetic lines of force. It seems that their motions are more influenced by the changes inside the main sunspots (the magnetic field rebuilding, the sunspot rotation, the Evershed effect inhomogeneities, etc.).

(3) The small umbrae and penumbra bits placed far from the main sunspots move

Kiepenheuer (ed.), Structure and Development of Solar Active Regions, 211 213. I.A.U. 

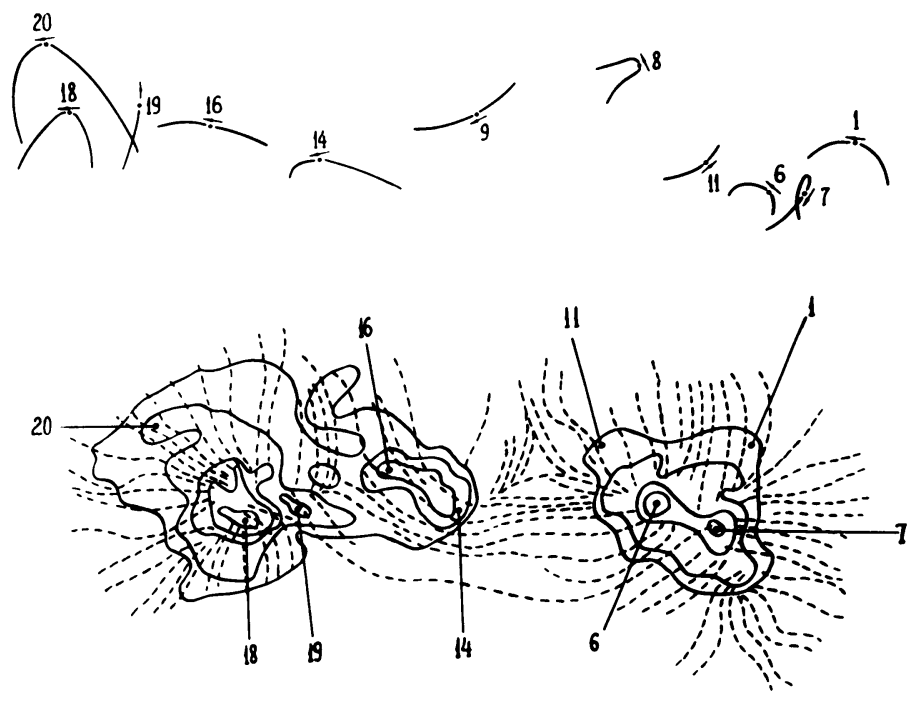

FIG. 1.
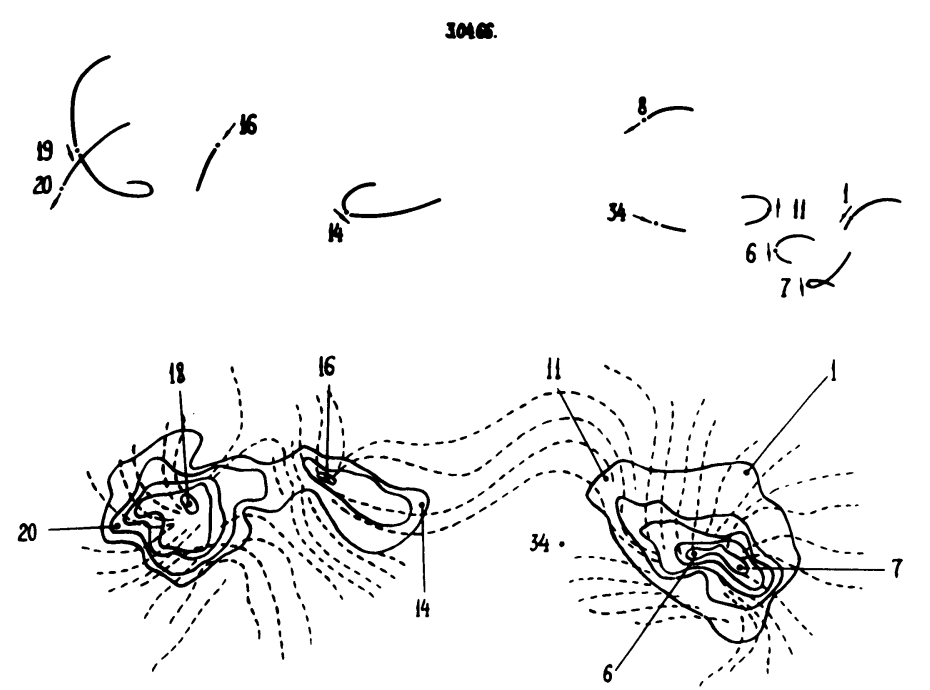

FIG. 2.

strongly along the magnetic lines of force, except for cases of close flares when it is impossible to determine that the cause of the line of force crossing by the sunspot is the magnetic-field rebuilding or the flare effect ('a shock wave').

Thus, if it is possible to make conclusions on the basis of such data we must suppose 
the following hierarchy of the dynamics (in the strict sense of the word) of matter in the active region:

(a) the main sunspot proper motions are caused by subphotospheric processes;

(b) the active region magnetic field is determined by the main sunspot magnetic fields and their proper motions;

(c) the small sunspot proper motions are corresponding to the active region magnetic-field structure.

\section{Acknowledgements}

I am grateful to Miss A.E. Syklen, Miss S. A. Radnaeva and Miss L. E. Vilutis for help in measurements, and to Dr. V.E. Stepanov for data on the magnetic fields.

\section{References}

Bumba, V. (1964) Prace Wroclawsk. towarz. nauk B, 112, 3-40.

Gopasyuk, S.I., Moreton, G. (1965) Report at the Solar Study Commis. Plenum (KISO) in Kislovodsk.

Kuklin, G.V., Syklen, A. E. (1966) Rezultaty nabl. i issled. $v$ period MGSS, 1, Moskva, p. 64.

\section{DISCUSSION}

H. U. Schmidt: I do not see that the horizontal motions across the magnetic field which you deduce from changes in the flux distribution with time contradict the magnetohydrodynamic theory. Quite to the contrary, it seems to me that you successfully apply a fundamental result of this theory, i.e. the frozen-in field principle for a plasma of high conductivity. This principle can be used to deduce a detailed horizontal flow field even if one has no directly identifiable spots in subsequent magnetic flux charts. I comment on this in the written version of my paper.

Kuklin: Firstly I compared the sunspot motions with the transversal magnetic field without considering any changes of the flux distribution in time. Secondly I said the motions of small sunspots and penumbra bits across the magnetic field are in contradiction with the magnetohydrodynamic theory because of assuming the small sunspots and penumbra bits are not features with deep located bottoms and so their motions are controlled by the magnetic field of the high photospheric layers which is observable. 